Journal of the Operations Research

Society of Japan

Vol. 32, No. 3, September 1989

\title{
A GENERALIZED VERSION OF ONE MACHINE MAXIMUM LATENESS PROBLEM
}

\author{
Minoru Tada \\ Ryukoku University \\ Hiroaki Ishii Toshio Nishida \\ Osaka University
}

(Received July 25, 1988)

Abstract In usual scheduling problem, machine speed is considered to be constant. Here we assume that the machine speed is controllable for each job and consider the maximum lateness problem on the single machine. The objective is to determine an optimal schedule and optimal jobwise speed of the machine minimizing the total sum of costs associated with the maximum lateness and jobwise machine speed.

\section{Introduction}

We consider a generalization of a classical one machine maximum lateness problem. In this problem, machine speed is assumed to be not constant but controllable. That is, machine speed is changeable for each job. The objective is to determine an optimal schedule and optimal jobwise speed of the machine minimizing the total sum of costs associated with the maximum lateness and jobwise machine speed.

In Section 2, we formulate a generalized version of one machine maximum lateness problem. In section 3, we show how our problem can be solved and propose an algorithm informally. In Section 4, we give an numerical example of this problem.

\section{Problem Formulation}

First some notations used in this paper are defined. Each job $J_{j}(1 \leqq j \leqq n)$ has the due date $d_{j}$ and 'standard' processing time $t_{j}$ (i.e., processing time when machine speed is unit). Let $C_{j}$ be the completion time and $s^{\prime}{ }_{j}$ machine speed for each job $J_{j}$. For a convenience sake, we set $s_{j}=1 / s^{\prime}{ }_{j}$. We assume that the cost of machine speed is linear and defined to be $a_{j}{ }^{\prime}{ }_{j}$, where $a_{j}$ $(1 \leqq j \leqq n)$ is a positive constant. Further, let $d_{1} \leqq d_{2} \leqq \cdots \leqq d_{n}$ without any loss of generality. Using these notations, the maximum lateness $L_{\max }$ is $\max _{j}\left(C_{j}-d_{j}\right)$. We consider the following problem PO.

$$
\begin{aligned}
& \text { P0: minimize }\left(a_{0} L_{\max }+\sum_{j} \alpha_{j} s^{\prime}{ }_{j}\right) \\
& \text { subject to } s^{\prime}{ }_{j}>0,
\end{aligned}
$$


where $a_{0}$ is a positive constant. Let $p_{j}$ be the actual processing time of each job (i.e., $p_{j}=t_{j} s$ ), and set $\bar{a}_{j}=a_{j} t_{j}$. Then the problem $P O$ is equivalent to the following $\mathrm{P} 1$.

$$
\text { P1: minimize }\left(a_{0} L_{\max }+\Sigma_{j} \bar{a}_{j} / p_{j}\right)
$$

Let maximum lateness be $t$. Then we have the following inequalities,

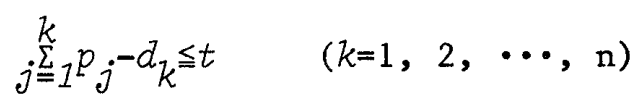

since we can assume the optimal processing order of jobs is $J_{1} \rightarrow J_{2} \rightarrow \cdots \rightarrow J_{n}[1]$. Thus, from (2.2) and (2.3), we have the following P2 which is equivalent to PO.

$$
\begin{aligned}
& \text { P2: minimize }\left(a_{0} t+\sum_{j=1}^{n} \bar{a}_{j} / p_{j}\right) \\
& \text { subject to } p_{j}>0, \\
& \qquad k_{j=1}^{k} p_{j} \leqq t+d_{k} \quad(k=1,2, \cdots, n)
\end{aligned}
$$

\section{Solving $P 2$}

To solve the problem $P 2$, we use the Lagrange method. Let $u_{j}(1 \leqq j \leqq n)$ be the Lagrange multiplier. Then $P 2$ is equivalent to the following problem:

$$
\max _{u \geqq 0} \min _{p} L(p, u),
$$

where

$$
\begin{aligned}
L(p, u) & =\sum_{j=1}^{n} \bar{a}_{j} / p_{j}+{ }_{j=1}^{n} u_{j}\left(\sum_{k=1}^{j} p_{k}-a_{j}-t\right) \\
& =\sum_{k=1}^{n}\left(\bar{a}_{k} / p_{k}+{ }_{j=k}^{n} u_{j} p_{k}\right)-\sum_{j=1}^{n} u_{j}\left(d_{j}+t\right) .
\end{aligned}
$$

Let $L(u)=\min _{p} L(p, u)$, then the inequality of arithmetic and geometric means

$$
\bar{a}_{k} / p_{k}+\sum_{j=k}^{n} u_{j} p_{k} \geq 2 \sqrt{\bar{a}_{k j} \sum_{k}^{n} u_{j}}
$$

leads to

$$
L(p, u) \geqq 2 \sum_{k=1}^{n} \sqrt{\bar{a}_{k} \sum_{j=k}^{n} u_{j}}-\sum_{j=1}^{n} u_{j} d_{j}-t_{j=1} \sum_{j}^{n}=L(u) .
$$

The equality in (3.3) is obtained, when

$$
p_{j}=\sqrt{\bar{a}_{j} / \sum_{\underline{=} j}^{n} u_{k}}
$$

Further, let $y_{k}=\sqrt{\sum_{j=k}^{n} u_{j}}$, i.e.,

$$
y_{k}{ }^{2}=\sum_{j=k}^{n} u_{j},
$$

or 


$$
\begin{aligned}
& y_{k}{ }^{2}-y_{k+1}{ }^{2}=u_{k} \geqq 0, \quad(k=1, \cdots, \mathrm{n}-1) \\
& y_{n}{ }^{2}=u_{n} \geqq 0 .
\end{aligned}
$$

Using these notations, $L(u)$ becomes the following function of $y$.

$$
\begin{aligned}
L(u)= & 2 \sum_{k=1}^{n} \sqrt{\bar{a}_{k}} y_{k}-{ }_{k=1}^{n-1}\left(y_{k}{ }^{2}-y_{k+1}{ }^{2}\right)\left(d_{k}+t\right)-y_{n}{ }^{2}\left(d_{n}+t\right) \\
= & 2 \sum_{k=1}^{n} \sqrt{\bar{a}_{k}} y_{k}-y_{1}^{2}\left(d_{1}+t\right)-\sum_{k=2}^{n} y_{k}{ }^{2}\left(d_{k}-d_{k-1}\right) \\
= & -\sum_{k=2}^{n}\left(d_{k}-d_{k-1}\right)\left(y_{k}-\sqrt{\bar{a}_{k}} /\left(d_{k}-d_{k-1}\right)\right)^{2} \\
& -\left(d_{1}+t\right)\left(y_{1}-\sqrt{\bar{a}_{1}} /\left(d_{1}+t\right)\right)^{2} \\
& +\sum_{k=2}^{n} \bar{a}_{k} /\left(d_{k}-d_{k-1}\right)+\bar{a}_{1} /\left(d_{1}+t\right) \triangleq_{G(y) .}
\end{aligned}
$$

Now, $e_{k}(1 \leqq k \leqq n)$ is defined as follows:

$$
e_{k}=A_{k} / B_{k}
$$

where

$$
\begin{aligned}
& A_{k}=\sqrt{\bar{a}_{k}} \quad \text { for } k=1,2, \cdots, \mathrm{n} \\
& B_{1}=d_{1}+t, \\
& B_{k}=d_{k}-d_{k-1} \quad \text { for } k=2, \cdots, \mathrm{n}
\end{aligned}
$$

Then the equation(3.6) is

$$
L(u)=-\sum_{k}^{n} B_{k}\left(y_{k}-e_{k}\right)^{2}+{ }_{k} \sum_{1}^{n} A_{k}^{2} / B_{k}=G(y) .
$$

We have following theorems.

Theorem 1. (i) If $e_{k} \geqq e_{k+1}$ for $k=1, \cdots, n-1$, then by setting $y_{j}=e_{j}$ (for $j=1, \cdots, \mathrm{n}$ ), an optimal value $f(t)$ (i.e., $f(t)=\max _{u \geqq 0} L(u)$ ) is obtained as follows :

$$
f(t)=\sum_{k=1}^{n} A_{k}^{2} / B_{k}
$$

(ii) If there exists an index $i$ such that $e_{i}<e_{i+1}$, an optimal solution $y$ giving $f(t)$ satisfies the condition $y_{i}=y_{i+1}$.

Proof: Since the case (i) is clear from (3.5) and (3.9), in order to show (ii), we prove that $y$ satisfying $y_{i}>y_{i+1}$ does not give $f(t)$. (a) $y_{i}>y_{i+1} \geqq e i+1$

As $y_{i}$ tends to $e_{i+1}$, the value of $G(\cdot)$ increases. Thus, the solution is obtained by setting $y_{i}=y_{i+1}$.

(b) $y_{i}>e_{i+1} \geqq y_{i+1}$

As $y_{i}$ and $y_{i+1}$ tend to $e_{i}$ and $e_{i+1}$, respectively, the value of $G(\cdot)$ increases. Similarly, the remaining two cases (c) $e_{i+1}>y_{i} \geqq e_{i}>y_{i+1}$, (d) $e_{i} \geqq y_{i}>$ $y_{i+1}$ may be proved.

Q.E.D. 
Theorem 2. If $e_{i}<e_{i+1}$ for some $i(2 \leqq i \leqq n-1)$, then setting as $y_{i}=y_{i+1}$ and $e_{i}=\left(A_{i}+A_{i+1}\right) /\left(B_{i}+B_{i+1}\right)$ is valid.

Proof: Since validity of setting as $y_{i}=y_{i+1}$ is clear from Theorem 1 , we prove that $e_{i}=\left(A_{i}+A_{i+1}\right) /\left(B_{i}+B_{i+1}\right)$. Now it is sufficient to take out terms associated with only the index $i$ and $i+1$ from (3.6). That is,

$$
\begin{aligned}
& 2 \sqrt{\bar{a}_{i}} y_{i}+2 \sqrt{a_{i+1}} y_{i+1}-y_{i}{ }^{2}\left(d_{i}-d_{i-1}\right)-y_{i+1}{ }^{2}\left(a_{i+1}-d_{i}\right) \\
&=-\left(d_{i+1}-a_{i-1}\right)\left(y_{i}-\left(\sqrt{\bar{a}_{i}}+\sqrt{\bar{a}_{i+1}}\right) /\left(d_{i+1}-d_{i-1}\right)\right)^{2} \\
&+\left(\bar{a}_{i}+\sqrt{\bar{a}_{i+1}}\right)^{2} /\left(d_{i+1}-d_{i-1}\right) \\
&=-\left(B_{i}+B_{i+1}\right)\left(y_{i}-\left(A_{i}+A_{i+1}\right) /\left(B_{i}+B_{i+1}\right)\right)^{2} \\
&+\left(A_{i}+A_{i+1}\right)^{2} /\left(B_{i}+B_{i+1}\right) .
\end{aligned}
$$

Accordingly, an optimal value $f(t)$ is obtained by setting as $y_{i}=y_{i+1}$ and $e_{i}=\left(A_{i}+A_{i+1}\right) /\left(B_{i}+B_{i+1}\right)$.

Q.E.D.

Corollary 1. Theorem 2 holds for $i=1$. That is, if $e_{1}<e_{2}$, then we can set $y_{1}=y_{2}$ and $e_{1}=\left(A_{1}+A_{2}\right) /\left(B_{1}+B_{2}\right)$.

The above theorems suggest an algorithm to solve the problem P2. First, compute $e_{j}$ for $j=2, \cdots$, n. If the case (ii) of the theorem 1 occurs (i.e., $e_{i}<e_{i+1}$ for some $i$ ), update $y_{j}, e_{j}$ as follows (Otherwise, go to the next step without executing the revision.):

$$
\begin{aligned}
& y_{j}=y_{j}, e_{j}=e_{j}, \text { if } j=2, \cdots, i-1, \\
& y_{i}=y_{i+1}, e_{i}=\left(A_{i}+A_{i+1}\right) /\left(B_{i}+B_{i+1}\right), \text { if } j=i, \\
& y_{j}=y_{j+1}, e_{j}=e_{j+1}, \text { if } j=i+1, \cdots, n-1,
\end{aligned}
$$

and further, update $A_{j}, B_{j}$ as follows:

$$
\begin{aligned}
& A_{j}=A_{j}, B_{j}=B_{j}, \text { if } j=1, \cdots, i-1, \\
& A_{i}=A_{i}+A_{i+1}, B_{i}=B_{i}+B_{i+1}, \text { if } j=i, \\
& A_{j}=A_{j+1}, B_{j}=B_{j+1}, \text { if } j=i+1, \cdots, n-1, \\
& n=n-1 .
\end{aligned}
$$

After the above revision, test $e_{j}$ for the case (i) of the theorem. If the case ( $i i)$ is happened again, the same revision is repeated. Since the times of revision is at most $(n-1)$, the case ( $i)$ certainly occurs, and then the optimal value $f(t)$ is obtained. Next, by the following step, check whether $e_{1}$ $\geqq e_{2}$ or not. If $e_{1} \geqq e_{2}$, that is

$$
t \leqq \sqrt{\bar{a}_{1}} / e_{2}-a_{1},
$$


then set $y_{1}=e_{1}, y_{2}=e_{2}, \cdots, y_{q}=e_{q}$, where $q$ is an integer that is less than or equal to $(n-1)$. Then the shape of objective function in the range (3.12) is

$$
\left(a_{0} t+\text { const. }+\bar{a}_{1} /\left(d_{1}+t\right)\right)=F(t) \text {. }
$$

Since the differential of $F(t)$ is

$$
\mathrm{d} F(t) / \mathrm{d} t=a_{0}-\bar{a}_{1} /\left(a_{1}+t\right)^{2},
$$

we obtain the optimal solution as follows:

$$
t=\sqrt{a_{1} / a_{0}}-a_{1}
$$

Otherwise (i.e., $e_{1}<e_{2}$ ), then reset $e_{1}$ as follows:

$$
\begin{aligned}
& y_{1}=y_{2}, e_{1}=\left(A_{1}+A_{2}\right) /\left(B_{1}+B_{2}\right), \\
& y_{j}=y_{j+1}, e_{j}=e_{j+1}, \text { if } j=2, \cdots, q .
\end{aligned}
$$

If $e_{1}<e_{2}$ is occurs again, then the same revision is repeated. Since $e_{1} \geqq e_{2}$ is certainly occurs after at most $(q-1)$ revisions, we obtain eventually the objective function $F(t)$ to be minimized is determined. And then, since each optimal value of $u_{j}$ is decided, the optimal processing time is obtained from $(3.4)$.

Theorem 3. The optimal value $y^{*}$ of $y_{1}$ is equal to $\sqrt{a_{0}}$. Thus, inequality $e_{1} \geqq e_{2}$ is equivalent to $\sqrt{a_{0}} \geqq e_{2}$, in other wards, $e_{1}=\sqrt{a_{0}}$.

Proof: First, we prove the case without updating $e_{1}$. By definition, $e_{1}=A_{1} /\left(d_{1}+t\right)$. Also, from (3.14), $t=A_{1} / \sqrt{a_{0}}-d_{1}$. Thus,

$$
e_{1}=A_{1} /\left(d_{1}+A_{1} / \sqrt{a_{0}}-d_{1}\right)=\sqrt{a_{0}}
$$

Next, we consider the case after executing the revision (3.15). Then, the shape of the objective function is

$$
F(t)=\left(a_{0} t+\text { const. }+\left(A_{1}+A_{2}\right)^{2} /\left(d_{1}+t+B_{2}\right)\right)
$$

Setting $\mathrm{d} F(t) / \mathrm{d} t=0$, we obtain $t=\left(A_{1}+A_{2}\right) / \sqrt{a_{0}}-\left(d_{1}+B_{2}\right)$. Using this value of in the updated expression

$$
e_{1}=\left(A_{1}+A_{2}\right) /\left(B_{1}+B_{2}\right)=\left(A_{1}+A_{2}\right) /\left(t+d_{1}+B_{2}\right)
$$

we obtain $e_{1}=\sqrt{a_{0}}$.

Q.E.D.

Since details of the solution procedure are complicated, we illustrate it by an example in the next section.

\section{An Example}

We consider an example in order to show the procedure obtaining the solu- 
tion. The number of jobs is five and their triplets $T_{j}=\left(t_{j}, d_{j}, a_{j}\right)$ are as follows:

$$
T_{1}=(5,1,3), T_{2}=(4,4,2), T_{3}=(3,4,5), T_{4}=(3,5,1), T_{5}=(5,9,2) \text {. }
$$

Also, let $a_{0}=4$. First, $e_{j}(j=2, \cdots, 5)$ are calculated from (3.7) and (3.8):

$$
e_{2}=\sqrt{8 / 3}=0.943, e_{3}=\sqrt{15} / 0=\infty, e_{4}=\sqrt{3} / 1=1.732, e_{5}=\sqrt{10} / 4=0.791
$$

The case (ii) of theorem 1 is happened, since $e_{2}<e_{3}$. Accordingly, update $y_{j}$, $e_{j}$ from (3.11) as follows:

$$
\begin{aligned}
& y_{2}=y_{3}, \quad e_{2}=(\sqrt{8}+\sqrt{15}) /(3+0)=2.234, \\
& y_{3}=y_{4}, \quad e_{3}=e_{4}=\sqrt{3}=1.732, \\
& y_{4}=y_{5}, \quad e_{4}=e_{5}=0.791 .
\end{aligned}
$$

$$
A_{2}=\sqrt{8}+\sqrt{15}, B_{2}=3+0, A_{3}=\sqrt{3}, B_{3}=1, A_{4}=\sqrt{10}, B_{4}=4 \text {. }
$$

Then, the case (i) occurs, that is, $e_{2} \geqq \cdots \geqq e_{5}$.

Next, check whether $e_{1} \geqq e_{2}$ or not. From theorem $3, e_{1} \geqq e_{2}$ is equivalent to $\sqrt{a_{0}} \geqq e_{2}$. Thus, we check $\sqrt{a_{0}} \geqq e_{2}$. Since $\sqrt{a_{0}}=2<2.234=e_{2}$, the revision (3.15) is executed:

$$
\begin{aligned}
& y_{1}=y_{2}, \quad e_{1}=\left(A_{1}+A_{2}\right) /\left(B_{1}+B_{2}\right), \\
& y_{2}=y_{3}, \quad e_{2}=e_{3}=1.732, \\
& y_{3}=y_{4}, \quad e_{3}=e_{4}=0.791 .
\end{aligned}
$$

$$
A_{1}=\sqrt{15}+\sqrt{8}+\sqrt{15}, B_{1}=t+4, A_{2}=\sqrt{3}, B_{2}=1, A_{3}=\sqrt{10}, B_{3}=4 \text {. }
$$

Now the case (i) occurs since $\sqrt{a_{0}}=2 \geqq 1.732=e_{2}$. Thus, we compute $t$ as follows:

$$
t=A_{1} / \sqrt{a_{0}}-D_{k}=(\sqrt{15}+\sqrt{8}+\sqrt{15}) / 2-4=1.287,
$$

where $D_{k}=B_{1}-t$. In this case, $D_{k}=d_{3}$. Each optimal solution is obtained as follows :

$$
\begin{aligned}
& y_{1}=y_{2}=y_{3}=e_{1}=\sqrt{a_{0}}=2, \\
& y_{4}=e_{2}=1.732, y_{5}=e_{3}=0.791 .
\end{aligned}
$$

Next Figure 1 illustrates flow of our solution procedure for this example.

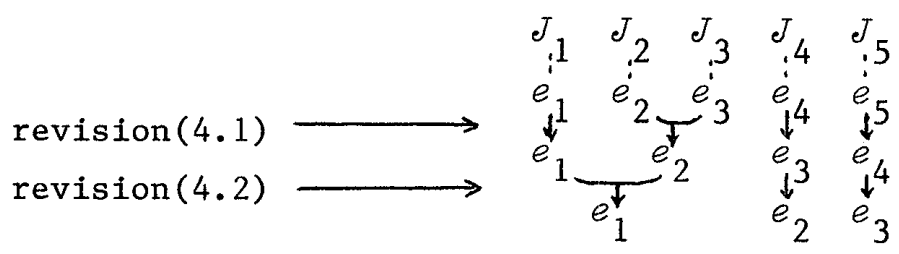

Fig. 1 Flow of the solution procedure 
Thus, the optimal processing time is obtained from (3.4),

$$
\begin{aligned}
& p_{1}=\sqrt{15} / 2=1.936, p_{2}=\sqrt{8} / 2=1.414, p_{3}=\sqrt{15} / 2=1.936, \\
& p_{4}=\sqrt{3} / 1.732=1, p_{5}=\sqrt{10} / 0.791=3.998
\end{aligned}
$$

Accordingly, each optimal speed $\left(s_{j}{ }^{\prime}=t_{j} / p_{j}\right)$ is decided as follows:

$$
\begin{aligned}
& s_{1}^{\prime}=5 / 1.936=2.583, s_{2}^{\prime}=4 / 1.414=2.829, \\
& s_{3}{ }^{\prime}=3 / 1.936=1.550, s_{4}^{\prime}=3 / 1=3, s_{5}{ }^{\prime}=5 / 3.998=1.251 .
\end{aligned}
$$

Theorem 4. The solution procedure illustrated by the above example requires $O(n \log n)$ computational time.

Proof: Each computational time of the procedure is shown as follows:

(a) Sorting of due dates $d_{j}: 0(n \log n)$.

(b) Constraction of $e_{j}(j \geqq 2): 0(n-1)$.

(c) Revision of $e_{j}(j \geqq 1): 0(n)$,

that is, finding $e_{2}$ such that $e_{2} \leqq \sqrt{a_{0}}$ requires $O(n-1)$ comparisons, and updating of $e_{j}$ is $0(n)$.

(d) Remaining operations: $0(n)$.

Therefore (a) dominates others and so $0(n \log n)$ computational time is required.

Q.E.D.

Reference

[1] W.E.Smith: Various Optimizer for Single Stage Production. Nav. Res. Log. Quart., Vo1.3 (1956), 59-66.

\author{
Minoru TADA: Faculty of Business Admin- \\ istration, Ryukoku University, \\ Fukakusa, Fushimi-ku, Kyoto 612, Japan.
}

